University of Nebraska - Lincoln

DigitalCommons@University of Nebraska - Lincoln

Faculty Publications, Department of Physics and Astronomy

Research Papers in Physics and Astronomy

2-13-2004

\title{
Nondipole Effects in Photo-Double-Ionization of He by a vuv Photon
}

Andrei Y. Istomin

University of Nebraska-Lincoln, aistomin2@unl.edu

N. L. Manakov

Voronezh State University, manakov@phys.vsu.ru

A. V. Meremianin

University of Freiburg, meremianin@phys.vsu.ru

Anthony F. Starace

University of Nebraska-Lincoln, astarace1@unl.edu

Follow this and additional works at: https://digitalcommons.unl.edu/physicsfacpub

Part of the Physics Commons

Istomin, Andrei Y.; Manakov, N. L.; Meremianin, A. V.; and Starace, Anthony F., "Nondipole Effects in PhotoDouble-Ionization of He by a vuv Photon" (2004). Faculty Publications, Department of Physics and Astronomy. 39.

https://digitalcommons.unl.edu/physicsfacpub/39

This Article is brought to you for free and open access by the Research Papers in Physics and Astronomy at DigitalCommons@University of Nebraska - Lincoln. It has been accepted for inclusion in Faculty Publications, Department of Physics and Astronomy by an authorized administrator of DigitalCommons@University of Nebraska Lincoln. 


\title{
Nondipole Effects in Photo-Double-Ionization of He by a vuv Photon
}

\author{
Andrei Y. Istomin, ${ }^{1}$ N. L. Manakov, ${ }^{2}$ A.V. Meremianin, ${ }^{3}$ and Anthony F. Starace ${ }^{1}$ \\ ${ }^{1}$ Department of Physics and Astronomy, The University of Nebraska, Lincoln, Nebraska 68588-0111, USA \\ ${ }^{2}$ Physics Department, Voronezh State University, Voronezh 394006, Russia \\ ${ }^{3}$ Theoretische Quantendynamik, Fakultät für Physik, University of Freiburg, Freiburg, D-79104 Germany
}

(Received 26 September 2003; published 13 February 2004)

\begin{abstract}
We present a dynamical model-independent, ab initio parametrization of the quadrupole transition amplitude for photo-double-ionization of He. An asymmetry of the triple differential cross section induced by the nondipole corrections is discussed and shown to be significant even for an excess energy as low as $80 \mathrm{eV}$. We provide predictions for two different kinds of experiments in which nondipole effects should be observable with current experimental capabilities.
\end{abstract}

PACS numbers: $32.80 . \mathrm{Fb}$

In recent years two major themes in studies of atomic photoionization have been the analysis of nondipole (or retardation) effects in single-electron photoionization (SPI) (by x-ray and vuv photons) and the analysis of photo-double-ionization (PDI), especially in the vuv range of photon energies. Although the first nondipole SPI measurements for Ar were in the x-ray region [1], very recently the forward-backward asymmetry of the photoelectron angular distributions (i.e., with respect to the photon wave vector $\mathbf{k}$, originating from the interference of dipole and quadrupole SPI amplitudes) has been found to be significant also in the vuv region (see, e.g., results for Xe at $E_{\gamma} \lesssim 200 \mathrm{eV}$ [2] and for $\mathrm{He}$ at $E_{\gamma} \lesssim$ $160 \mathrm{eV}$ [3]). Besides the asymmetry in the angular distributions, nondipole effects lead also to new features in SPI for spin-resolved photoelectron measurements [4] and for the case of polarized atoms [5].

The process of PDI has attracted much interest in view of its importance for analyzing electron correlations (for recent reviews, see [6,7]). Most experiments concern the $\mathrm{He}$ atom, in which PDI represents the prototype for threebody fragmentation of a Coulomb system by a single photon. The photon energies employed typically range between 100 and $530 \mathrm{eV}$. Measurements of the triple differential cross section (TDCS) at these energies have been interpreted using the electric-dipole approximation. Recent advances in experimental techniques allow the measurement of even very small TDCSs of the order of $\left(10^{-2}-10^{-3}\right) \mathrm{beV}^{-1} \mathrm{sr}^{-2}$ [8].

Owing to the importance of nondipole effects in SPI, it is somewhat surprising that there are no analyses of these effects for PDI TDCSs in the vuv region [9]. Presumably, this is due to two circumstances. First, even for the case of dipole PDI an accurate account of electron correlations requires time-consuming numerical techniques that have been developed only relatively recently. In addition, for the case of two ejected electrons with asymptotic momenta $\mathbf{p}_{1}$ and $\mathbf{p}_{2}$, the analysis of the kinematical part of the problem, i.e., the separation of kinematical and dynamical factors in the TDCS, is much more complicated than for SPI and even in the dipole case requires the use of advanced angular momentum techniques $[6,10]$. Second, existing experimental data do not exhibit significant deviations from theoretical predictions based on the electric-dipole approximation. However, nearly all TDCS measurements were performed in the perpendicular plane geometry, with the photoelectron detection plane orthogonal to the direction of the photon beam. (The only exceptions, to our knowledge, are the measurements in Ref. [11] and in more recent work [12], all of which deal with low excess energies, $\leq 25 \mathrm{eV}$.) In lowest order, retardation corrections stem from terms $\sim(\mathbf{k} \cdot \mathbf{r})$ in the power series expansion of the vector potential. Therefore, they can enter the PDI amplitude and the TDCS only through scalar products $\left(\mathbf{k} \cdot \mathbf{p}_{1}\right)$ and $\left(\mathbf{k} \cdot \mathbf{p}_{2}\right)$, which obviously vanish for the case of perpendicular plane geometry [13].

In this Letter we derive a general parametrization for the PDI amplitude of He taking into account the dipole $(E 1)$ and quadrupole (E2) components of the electronphoton interaction operator. We then discuss the nondipole effects to be expected in the TDCS (which originate from interference of the $E 1$ and $E 2$ amplitudes), such as the asymmetry with respect to the direction of the wave vector $\mathbf{k}$ of a linearly polarized photon. Finally, the results of our numerical calculations demonstrate that these effects are significant and may be observable even at excess energies as low as $80 \mathrm{eV}$.

The PDI amplitude $A$, taking into account retardation corrections in lowest order is

$$
A=A_{d}+A_{q}=\left\langle\mathbf{p}_{1} \mathbf{p}_{2}\left|(\mathbf{e} \cdot \mathbf{D})+\left(\{\mathbf{e} \otimes \hat{\mathbf{k}}\}_{2} \cdot Q_{2}\right)\right| 0\right\rangle
$$

where $\left|\mathbf{p}_{1} \mathbf{p}_{2}\right\rangle$ is the final state, and $\mathbf{e}$ is the unit (generally complex, $\mathbf{e} \cdot \mathbf{e}^{*}=1$ ) photon polarization vector. Note that the magnetic dipole operator does not contribute for the case of an initial ${ }^{1} S_{0}$ state, $|0\rangle$. The operators $\mathbf{D}=\mathbf{r}_{1}+\mathbf{r}_{2}$ and $Q_{2 m}=(i / 2) \alpha \omega\left(\left\{\mathbf{r}_{1} \otimes \mathbf{r}_{1}\right\}_{2 m}+\left\{\mathbf{r}_{2} \otimes \mathbf{r}_{2}\right\}_{2 m}\right)$ in Eq. (1) correspond to dipole $\left(A_{d}\right)$ and quadrupole $\left(A_{q}\right)$ parts of the transition amplitude in the length gauge. [In the 
velocity gauge, the substitutions, $\mathbf{D} \rightarrow-i\left(\nabla_{1}+\nabla_{2}\right)$ and $Q_{2 m} \rightarrow \alpha \omega\left(\left\{\mathbf{r}_{1} \otimes \nabla_{1}\right\}_{2 m}+\left\{\mathbf{r}_{2} \otimes \nabla_{2}\right\}_{2 m}\right)$, should be made.] We use atomic units and standard notations for the irreducible tensor products [14]; $\alpha=1 / 137$.

In order to establish the dependence of the quadrupole amplitude $A_{q}$ on the photon parameters $\mathbf{e}$ and $\mathbf{k}$ and the mutual angle $\theta_{12} \equiv \theta$ between $\mathbf{p}_{1}$ and $\mathbf{p}_{2}$, we employ the techniques developed for parametrization of the electricdipole TDCS [10]. First, we use the well-known multipole expansion of the final state $\left|\mathbf{p}_{1} \mathbf{p}_{2}\right\rangle$ in terms of bipolar harmonics $C_{l m}^{l_{1} l_{2}}\left(\hat{\mathbf{p}}_{1}, \hat{\mathbf{p}}_{2}\right)$ of the unit vectors $\hat{\mathbf{p}}_{1}$ and $\hat{\mathbf{p}}_{2}$,

$$
\left|\mathbf{p}_{1} \mathbf{p}_{2}\right\rangle=\sum_{l_{1} l_{2} l m} C_{l m}^{l_{1} l_{2} *}\left(\hat{\mathbf{p}}_{1}, \hat{\mathbf{p}}_{2}\right)\left|p_{1} p_{2} ;\left(l_{1} l_{2}\right) l m\right\rangle,
$$

together with the Wigner-Eckart theorem [14] to present the polarization-angular dependence of $A_{q}$ in terms of a sum of scalar products of two rank-2 tensors:

$$
A_{q}=\frac{1}{\sqrt{5}} \sum_{l_{1} l_{2}}\left(\{\mathbf{e} \otimes \hat{\mathbf{k}}\}_{2} \cdot C_{2}^{l_{1} l_{2}}\left(\hat{\mathbf{p}}_{1}, \hat{\mathbf{p}}_{2}\right)\right)\left\langle p_{1} p_{2} ;\left(l_{1} l_{2}\right) 2\left\|Q_{2}\right\| 0\right\rangle,
$$

where $\left\langle p_{1} p_{2} ;\left(l_{1} l_{2}\right) 2\left\|Q_{2}\right\| 0\right\rangle$ is the reduced matrix element of the operator $Q_{2 m}$ between the initial ${ }^{1} S_{0}$ state, $|0\rangle$, and the $D$-wave component of the two-electron continuum state with individual angular momenta $l_{1}$ and $l_{2}=l_{1}$, $l_{1} \pm 2$. The bipolar harmonics of rank 2 in Eq. (3) reduce to combinations of derivatives of the Legendre polynomials $P_{l}(\cos \theta)$ and three rank-2 tensors $\left\{\hat{\mathbf{p}}_{i} \otimes \hat{\mathbf{p}}_{j}\right\}_{2 m}$ with $(i, j)=(1,1),(1,2)$, and $(2,2)$ (see Ref. [10]). After calculating the scalar products of these tensors with the tensor $\{\mathbf{e} \otimes \hat{\mathbf{k}}\}_{2 m}$ in Eq. (3), the amplitude $A_{q}$ may be presented in the following invariant form:

$$
\begin{aligned}
A_{q}= & g_{1}\left(\mathbf{e} \cdot \hat{\mathbf{p}}_{1}\right)\left(\hat{\mathbf{p}}_{1} \cdot \hat{\mathbf{k}}\right)+g_{2}\left(\mathbf{e} \cdot \hat{\mathbf{p}}_{2}\right)\left(\hat{\mathbf{p}}_{2} \cdot \hat{\mathbf{k}}\right) \\
& +g_{s}\left[\left(\mathbf{e} \cdot \hat{\mathbf{p}}_{1}\right)\left(\hat{\mathbf{p}}_{2} \cdot \hat{\mathbf{k}}\right)+\left(\mathbf{e} \cdot \hat{\mathbf{p}}_{2}\right)\left(\hat{\mathbf{p}}_{1} \cdot \hat{\mathbf{k}}\right)\right],
\end{aligned}
$$

in terms of two $\theta$-dependent functions: $g_{s} \equiv$ $g_{s}\left(p_{1}, p_{2}, \cos \theta\right)=g_{s}\left(p_{2}, p_{1}, \cos \theta\right)$ (which is symmetric in the arguments $p_{1}$ and $\left.p_{2}\right)$, and the function $g\left(p, p^{\prime}, \cos \theta\right)$, with $g_{1} \equiv g\left(p_{1}, p_{2}, \cos \theta\right) \quad$ and $\quad g_{2} \equiv$ $g\left(p_{2}, p_{1}, \cos \theta\right)$. The explicit forms of these functions are

$$
\begin{aligned}
g_{s}\left(p, p^{\prime}, \cos \theta\right) & =-\sum_{l=1}^{\infty}\left[\sum_{l^{\prime}=l \pm 2} Q_{l l^{\prime}}\left(p, p^{\prime}\right) P_{\left(l+l^{\prime}\right) / 2}^{(2)}(\cos \theta)+\sqrt{6} Q_{l l}\left(p, p^{\prime}\right)\left(P_{l+1}^{(2)}(\cos \theta)-\frac{2 l+3}{2} P_{l}^{(1)}(\cos \theta)\right)\right], \\
g\left(p, p^{\prime}, \cos \theta\right) & =\sum_{l=2}^{\infty}\left[\sum_{l^{\prime}=l \pm 2} Q_{l l^{\prime}}\left(p, p^{\prime}\right)+\sqrt{6} Q_{l l}\left(p, p^{\prime}\right)\right] P_{l}^{(2)}(\cos \theta),
\end{aligned}
$$

where $P_{l}^{(n)}(x)=\left(d^{n} / d x^{n}\right) P_{l}(x)$ and where

$$
Q_{l l^{\prime}}\left(p, p^{\prime}\right)=2(-1) l \sqrt{\frac{\left(l+l^{\prime}-2\right) !}{\left(l+l^{\prime}+3\right) !}}\left\langle p p^{\prime} ;\left(l l^{\prime}\right) 2\left\|Q_{2}\right\| 0\right\rangle .
$$

The similar parametrization of the dipole amplitude $A_{d}$, corresponding to Eqs. (4)-(6), is well known $[6,10]$,

$$
A_{d}=f_{1}\left(\mathbf{e} \cdot \hat{\mathbf{p}}_{1}\right)+f_{2}\left(\mathbf{e} \cdot \hat{\mathbf{p}}_{2}\right),
$$

where $f_{1} \equiv f\left(p_{1}, p_{2}, \cos \theta\right)$ and $f_{2} \equiv f\left(p_{2}, p_{1}, \cos \theta\right)$ are defined by a single function,

$$
f\left(p, p^{\prime}, \cos \theta\right)=\sum_{l=1}^{\infty}\left[\sum_{l^{\prime}=l \pm 1} D_{l l^{\prime}}\left(p, p^{\prime}\right)\right] P_{l}^{(1)}(\cos \theta),
$$

where

$$
D_{l l^{\prime}}\left(p, p^{\prime}\right)=(-1)^{l^{\prime}} \frac{\left\langle p p^{\prime} ;\left(l l^{\prime}\right) 1\|\mathbf{D}\| 0\right\rangle}{\sqrt{(2 l+1)\left(2 l^{\prime}+1\right) \max \left(l, l^{\prime}\right)}} .
$$

The results in Eqs. (4)-(9) are general and give $a b$ initio parametrizations of the dipole and quadrupole PDI amplitudes, independent of the dynamical model used for calculations of the reduced matrix elements (6) and (9). [Note that parametrization of the dipole amplitude $A_{d}$ in terms of the symmetrized amplitudes, $a_{g, u}=$ $\left(f_{1} \pm f_{2}\right) / 2$, equivalent to that in Eqs. (7)-(9), was obtained in Ref. [15].] Thus, the measurements of nondipole effects in PDI of He allow one to probe electron correlations in the $D$-wave part of the two-electron continuum state $\left|\mathbf{p}_{1} \mathbf{p}_{2}\right\rangle$ (since in the dipole PDI amplitude only the $P$-wave part contributes).

In order to estimate the magnitude of nondipole effects, we have used lowest-order perturbation theory (LOPT) in the interelectron interaction to calculate the dynamical factors $D_{l l^{\prime}}\left(p, p^{\prime}\right)$ and $Q_{l l^{\prime}}\left(p, p^{\prime}\right)$. As in Ref. [16], for excess energies of the order of tens of eV, final state electron correlations are taken into account perturbatively to lowest order, and the He ground state is represented by the variational (uncorrelated) wave function having the effective nuclear charge $Z=27 / 16$. This approach has been shown in Ref. [16] to provide predictions for the dipole TDCS that are in good agreement with absolute data and results of computationally intensive theoretical calculations for almost all mutual angles $\theta$ (with the only exception being the region of small $\theta, \theta \lesssim 60^{\circ}$ ). For an excess energy of $80 \mathrm{eV}$ our numerical calculations show that only individual photoelectron angular momenta $\left(l, l^{\prime}\right) \leq 6$ contribute significantly to $g_{s}$ and $g$, while only $\left(l, l^{\prime}\right) \leq 4$ contribute to $f$.

We restrict our analysis of the TDCS to the simplest and most important case of linearly polarized photons, $\mathbf{e} \equiv \hat{\boldsymbol{\epsilon}}$. For this case, the dipole-quadrupole TDCS,

$$
\frac{d \sigma}{d E_{1} d \Omega_{1} d \Omega_{2}} \equiv \sigma=\mathcal{A}\left|A_{d}+A_{q}\right|^{2},
$$

where $\mathcal{A}=4 \pi^{2} \alpha p_{1} p_{2} / \omega$, takes the following form upon neglecting the small $E 2-E 2$ terms: 


$$
\sigma=\sigma_{d}+\mathcal{A}\left[\alpha_{1}\left(\hat{\mathbf{p}}_{1} \cdot \hat{\mathbf{k}}\right)+\alpha_{2}\left(\hat{\mathbf{p}}_{2} \cdot \hat{\mathbf{k}}\right)\right]
$$

In this equation, $\sigma_{d}=\mathcal{A}\left|A_{d}\right|^{2}$ is the dipole TDCS, and the $\hat{\mathbf{k}}$-independent parameters $\alpha_{1}$ and $\alpha_{2}$ describe the dipole-quadrupole interference:

$$
\begin{aligned}
& \alpha_{1}=2 \operatorname{Re}\left\{A_{d}^{*}\left[g_{1}\left(\hat{\boldsymbol{\epsilon}} \cdot \hat{\mathbf{p}}_{1}\right)+g_{s}\left(\hat{\boldsymbol{\epsilon}} \cdot \hat{\mathbf{p}}_{2}\right)\right]\right\}, \\
& \alpha_{2}=2 \operatorname{Re}\left\{A_{d}^{*}\left[g_{2}\left(\hat{\boldsymbol{\epsilon}} \cdot \hat{\mathbf{p}}_{2}\right)+g_{s}\left(\hat{\boldsymbol{\epsilon}} \cdot \hat{\mathbf{p}}_{1}\right)\right]\right\} .
\end{aligned}
$$

The parameters $\alpha_{1}$ and $\alpha_{2}$ may be determined from two measurements employing a nonorthogonal geometry with different directions of the photon wave vector. As Eqs. (11) and (12) show, the E1-E2 interference is maximal when all four vectors, $\mathbf{k}, \hat{\boldsymbol{\epsilon}}, \mathbf{p}_{1}$, and $\mathbf{p}_{2}$ lie in one plane (the coplanar geometry). As follows from Eq. (11), the difference, $\Delta$, between two TDCSs measured for a fixed photon polarization axis (along $\hat{\boldsymbol{\epsilon}}$ ) but for different photon propagation directions, $\hat{\mathbf{k}}$ and $\hat{\mathbf{k}}^{\prime}$, i.e., $\Delta=\sigma(\hat{\mathbf{\epsilon}}, \hat{\mathbf{k}})-$ $\sigma\left(\hat{\mathbf{\epsilon}}, \hat{\mathbf{k}}^{\prime}\right)$, is

$$
\Delta=\mathcal{A}\left[\alpha_{1}\left(\hat{\mathbf{p}}_{1} \cdot\left[\hat{\mathbf{k}}-\hat{\mathbf{k}}^{\prime}\right]\right)+\alpha_{2}\left(\hat{\mathbf{p}}_{2} \cdot\left[\hat{\mathbf{k}}-\hat{\mathbf{k}}^{\prime}\right]\right)\right] .
$$

The quantity $|\Delta|$ has a maximum at $\hat{\mathbf{k}}^{\prime}=-\hat{\mathbf{k}}$, i.e., the maximum difference between two TDCSs occurs when the second measurement is done using a photon propagation direction $\hat{\mathbf{k}}^{\prime}=-\hat{\mathbf{k}}$ and the same polarization $\hat{\boldsymbol{\epsilon}}$. In Fig. 1 we propose two geometries suitable for experimental measurements of nondipole effects in the TDCS, for equal and unequal energy sharing, respectively.

For equal energy sharing [see Fig. 1(a)], the experiment involves two measurements of the TDCS as a function of angle $\theta_{2}$ for fixed angle $\theta_{1}$ : one with photon beam parameters $(\hat{\boldsymbol{\epsilon}}, \hat{\mathbf{k}})$, and another with $(\hat{\boldsymbol{\epsilon}},-\hat{\mathbf{k}})$, in which the direction of the photon beam is inverted (or, equivalently, the detection plane is rotated by an angle of $180^{\circ}$ about the photon polarization axis $\hat{\boldsymbol{\epsilon}})$. The difference between the two measured TDCSs is [cf. Eq. (13)]: $\Delta=$ $2 \mathcal{A}\left[\alpha_{1}\left(\hat{\mathbf{p}}_{1} \cdot \hat{\mathbf{k}}\right)+\alpha_{2}\left(\hat{\mathbf{p}}_{2} \cdot \hat{\mathbf{k}}\right)\right]$. We have calculated a set of TDCSs for the geometry shown in Fig. 1(a) for two values of the angle $\theta_{1}$ and for two opposite directions of the
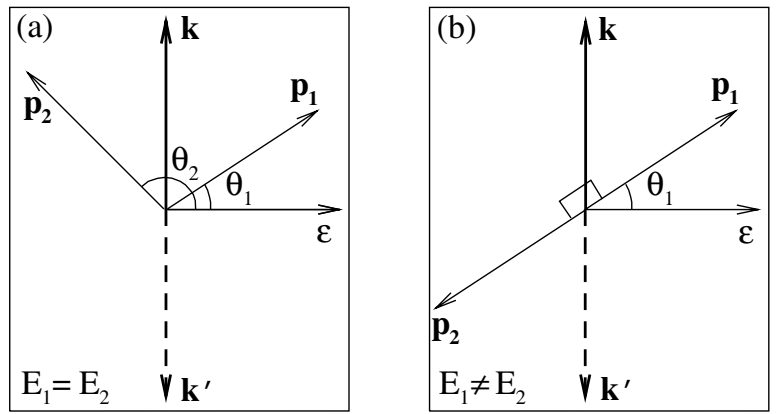

FIG. 1. Experimental geometries suitable for observation of nondipole effects in PDI. Vectors $\mathbf{p}_{1}, \mathbf{p}_{2}, \hat{\boldsymbol{\epsilon}}, \mathbf{k}$, and $\mathbf{k}^{\prime}$ lie in one plane. Two independent measurements of the TDCS should be performed: one for $(\hat{\boldsymbol{\epsilon}}, \mathbf{k})$, and another for $\left(\hat{\boldsymbol{\epsilon}}, \mathbf{k}^{\prime}=-\mathbf{k}\right)$. (a) Case of equal energy sharing; (b) case of unequal energy sharing (for $\hat{\mathbf{p}}_{2}=-\hat{\mathbf{p}}_{1}$, i.e., $\theta_{12} \equiv \theta=180^{\circ}$ ). photon momentum $\mathbf{k}$. The nondipole corrections (i.e., the magnitude of the parameter $\Delta$ ) are maximal for $\theta_{1} \lesssim$ $30^{\circ}$, although for $\theta_{1}=90^{\circ}$ the effect is sizable as well. Our numerical results for the TDCS and the parameters $\Delta$ and $\Delta_{\text {rel }}$, where

$$
\Delta_{\text {rel }}=[\sigma(\hat{\boldsymbol{\epsilon}}, \hat{\mathbf{k}})-\sigma(\hat{\boldsymbol{\epsilon}},-\hat{\mathbf{k}})] /[\sigma(\hat{\boldsymbol{\epsilon}}, \hat{\mathbf{k}})+\sigma(\hat{\boldsymbol{\epsilon}},-\hat{\mathbf{k}})]
$$

are presented in Fig. 2 for an excess energy of $80 \mathrm{eV}$ $\left(E_{\gamma}=159 \mathrm{eV}\right)$ as functions of the angle $\theta_{2}$ for two fixed values of $\theta_{1}$ : in Figs. 2(a)-2(c), $\theta_{1}=0^{\circ}$, and in Figs. 2(d) $-2(\mathrm{f}), \theta_{1}=15^{\circ}$. One sees that the difference between the two TDCSs at their maxima is approximately $15 \%-20 \%$ of the TDCS magnitude. The relative effect is even larger for $\theta_{12} \equiv \theta \approx 180^{\circ}$, although the TDCS is much smaller in this angular range since the dipole amplitude $A_{d}$ vanishes for back-to-back emission for equal energy sharing [17].

For unequal energy sharing, the back-to-back electron emission geometry is especially attractive [see Fig. 1(b)]. The appropriate experiment for this case involves two measurements of the TDCS as a function of the angle $\theta_{1}$ between the photon polarization $\hat{\boldsymbol{\epsilon}}$ and the photoelectron ejection axis, $\hat{\mathbf{p}}_{1}=-\hat{\mathbf{p}}_{2}$, with the photon beam directed along $\hat{\mathbf{k}}$ and $-\hat{\mathbf{k}}$, respectively. The difference between the TDCSs for these two experimental arrangements is
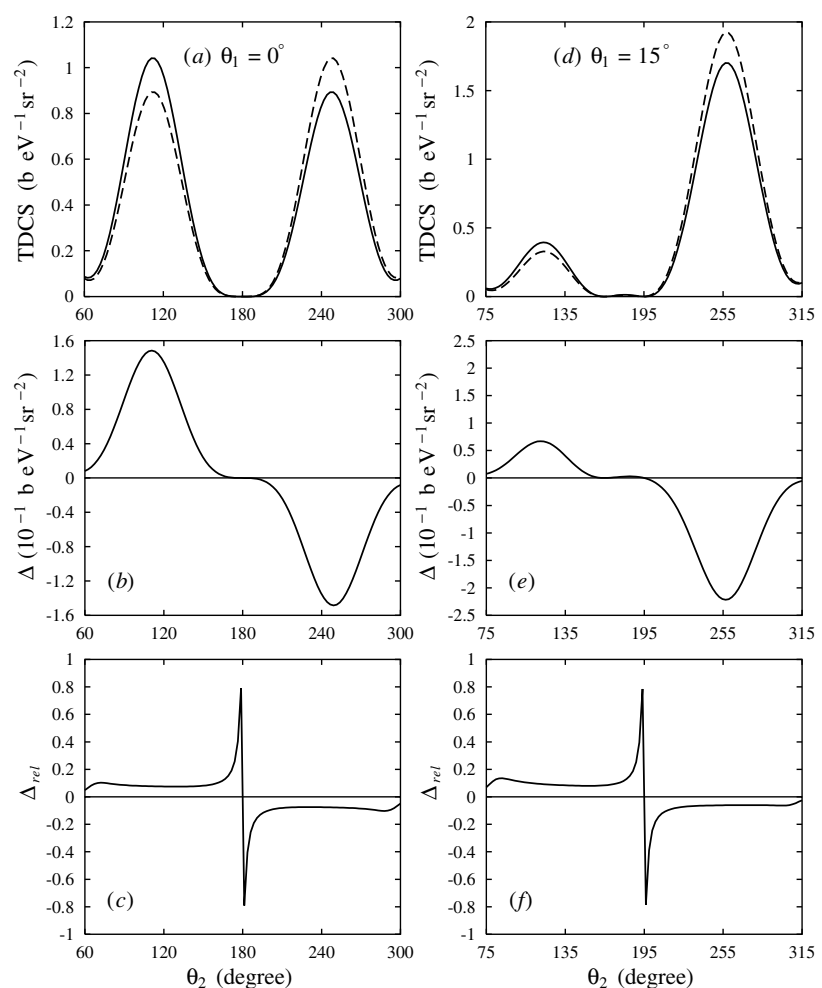

FIG. 2. Present results for the dipole-quadrupole TDCS [cf. Eqs. (10) and (11)] and parameters $\Delta$ and $\Delta_{\text {rel }}$ [cf. Eqs. (13) and (14)] for the PDI of He by linearly polarized photons for the geometry of Fig. 1(a) and an excess energy of $80 \mathrm{eV}$. In (a) and (d), full curves define $\sigma(\hat{\boldsymbol{\epsilon}}, \hat{\mathbf{k}})$; dashed curves define $\sigma(\hat{\boldsymbol{\epsilon}},-\hat{\mathbf{k}})$. In (a) $-(\mathrm{c}), \theta_{1}=0^{\circ}$; in (d)-(f), $\theta_{1}=15^{\circ}$. 

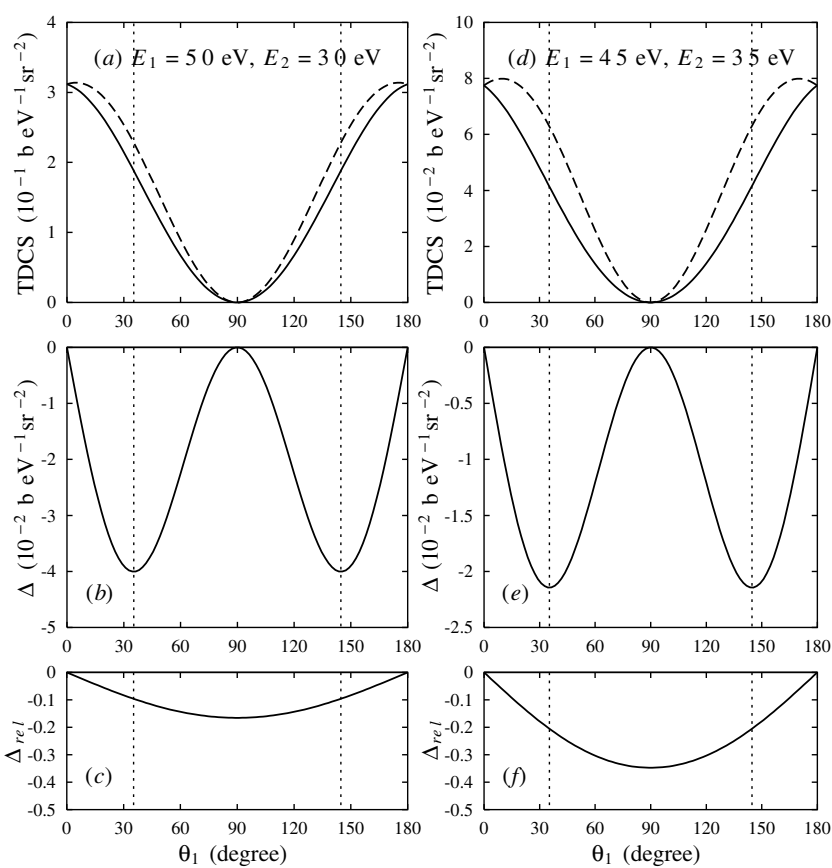

FIG. 3. The same as in Fig. 2, but for the back-to-back emission geometry shown in Fig. 1(b) for two unequal energy sharings [indicated in (a) and (d)]. The parameter $\Delta$ has maxima at $\theta_{1}=35.26^{\circ}$ and $\theta_{1}=144.7^{\circ}$, as shown by the dotted lines.

$$
\Delta=4 \mathcal{A}\left(\hat{\mathbf{p}}_{1} \cdot \hat{\mathbf{k}}\right)\left(\hat{\boldsymbol{\epsilon}} \cdot \hat{\mathbf{p}}_{1}\right)^{2} \operatorname{Re}\left[\left(f_{1}^{*}-f_{2}^{*}\right)\left(g_{1}+g_{2}-2 g_{s}\right)\right],
$$

where $\left(\hat{\mathbf{p}}_{1} \cdot \hat{\mathbf{k}}\right)\left(\hat{\boldsymbol{\epsilon}} \cdot \hat{\mathbf{p}}_{1}\right)^{2}=\sin \theta_{1} \cos ^{2} \theta_{1}$ for the geometry in Fig. 1(b). The maxima of this expression occur at $\theta_{1}=$ $\arcsin (1 / \sqrt{3}) \approx 35.26^{\circ}$ and $\theta_{1}=\pi-\arcsin (1 / \sqrt{3}) \approx$ $144.7^{\circ}$. Our LOPT results for the back-to-back emission geometry are presented in Fig. 3 for an excess energy of $80 \mathrm{eV}$ and two energy sharings: $E_{1}=50 \mathrm{eV}, E_{2}=30 \mathrm{eV}$; and $E_{1}=45 \mathrm{eV}, E_{2}=35 \mathrm{eV}$. As can be seen in Fig. 3, the nondipole effects are relatively large for nearly equal energy sharing since for this case the dipole amplitude $A_{d}$ is small. On the contrary, for more asymmetric energy sharings (e.g., $E_{1}=70 \mathrm{eV}$ and $E_{2}=10 \mathrm{eV}$ ) we find that the relative importance of the quadrupole corrections decreases (since $A_{d}$ is large compared to $A_{q}$ ).

In conclusion, our analysis shows that nondipole effects in PDI of He are sizable even at an excess energy as low as $80 \mathrm{eV}$ and should be observable in experiments using the coplanar geometry with current state-of-the-art capabilities. The analysis of nondipole effects in PDI by circularly polarized photons will be presented elsewhere.

This work was supported in part by the U.S. Department of Energy, Office of Science, Division of Chemical Sciences, Geosciences, and Biosciences, under Grant No. DE-FG03-96ER14646, by the joint Grant No. VZ-010-0 of the CRDF and the RF Ministry of Education (N. L. M.), and by the DFG in SFB 276 (A. V. M.).
[1] B. Krässig, M. Jung, D. S. Gemmell, E. P. Kanter, T. LeBrun, S. H. Southworth, and L. Young, Phys. Rev. Lett. 75, 4736 (1995).

[2] O. Hemmers, R. Guillemin, E. P. Kanter, B. Krässig, D.W. Lindle, S. H. Southworth, R. Wehlitz, J. Baker, A. Hudson, M. Lotrakul, D. Rolles, W. C. Stolte, I. C. Tran, A. Wolska, S.W. Yu, M. Ya. Amusia, K. T. Cheng, L. V. Chernysheva, W. R. Johnson, and S. T. Manson, Phys. Rev. Lett. 91, 053002 (2003).

[3] E. P. Kanter, B. Krässig, S. H. Southworth, R. Guillemin, O. Hemmers, D.W. Lindle, R. Wehlitz, M. Ya. Amusia, L. V. Chernysheva, and N. L. S. Martin, Phys. Rev. A 68 , 012714 (2003).

[4] N. A. Cherepkov and S. K. Semenov, J. Phys. B 34, L211 (2001); T. Khalil, B. Schmidtke, M. Drescher, N. Müller, and U. Heinzmann, Phys. Rev. Lett. 89, 053001 (2002).

[5] A. N. Grum-Grzhimailo, J. Phys. B 34, L359 (2001).

[6] J.S. Briggs and V. Schmidt, J. Phys. B 33, R1 (2000).

[7] G. C. King and L. Avaldi, J. Phys. B 33, R215 (2000).

[8] A. Knapp, A. Kheifets, I. Bray, Th. Weber, A. L. Landers, S. Schössler, T. Jahnke, J. Nickles, S. Kammer, O. Jagutzki, L. Ph. H. Schmidt, T. Osipov, J. Rösch, M. H. Prior, H. Schmidt-Böcking, C. L. Cocke, and R. Dörner, Phys. Rev. Lett. 89, 033004 (2002).

[9] To the best of our knowledge, only in the paper by M. A. Kornberg and J. E. Miraglia [Phys. Rev. A 52, 2915 (1995)] is the treatment of retardation effects for PDI of He by 1 and $3 \mathrm{keV}$ photons presented. However, these authors treat only double-differential cross sections, so that the angular dependences of their predicted cross sections are similar to those for SPI and are thus less sensitive to nondipole effects than are the TDCSs presented here.

[10] N. L. Manakov, S. I. Marmo, and A.V. Meremianin, J. Phys. B 29, 2711 (1996).

[11] A. Huetz, P. Lablanquie, L. Andric, P. Selles, and J. Mazeau, J. Phys. B 27, L13 (1994); P. Lablanquie, J. Mazeau, L. Andric, P. Selles, and A. Huetz, Phys. Rev. Lett. 74, 2192 (1995).

[12] D. P. Seccombe, S. A. Collins, T. J. Reddish, P. Selles, L. Malegat, A. K. Kazansky, and A. Huetz, J. Phys. B 35, 3767 (2002).

[13] Note that beyond-quadrupole terms do contribute in the perpendicular plane geometry, but existing experiments for He do not show any signatures of these corrections, which are of order $\alpha \omega$ as compared to E2.

[14] D. A. Varshalovich, A. N. Moskalev, and V. K. Khersonskii, Quantum Theory of Angular Momentum (World Scientific, Singapore, 1988).

[15] L. Malegat, P. Selles, and A. Huetz, J. Phys. B 30, 251 (1997).

[16] A. Y. Istomin, N. L. Manakov, and A. F. Starace, J. Phys. B 35, L543 (2002); Phys. Rev. A (to be published).

[17] Note that if one neglects $E 2-E 2$ terms, $\Delta_{\text {rel }}$ in Eq. (14) has a singularity when $A_{d}$ is zero. For this reason, our numerical results for $\Delta_{\text {rel }}$ include the $E 2-E 2$ terms in Eqs. (10) and (14), so that $\Delta_{\text {rel }}$ is finite for $\theta_{2}=180^{\circ}$ and $\theta_{2}=195^{\circ}$ in Fig. 2. 